\title{
Die NATO und der Klimawandel: Im Spannungsfeld zwischen politischen Erwartungen und militärischen Notwendigkeiten
}

https://doi.org/10.1515/sirius-2021-4009

\section{Einleitung}

Auf dem nächsten Gipfeltreffen der Staats- und Regierungschefs der NATO im Sommer 2022 will das Bündnis ein neues Strategisches Konzept verabschieden. Dieses würde das Konzept von 2010, dessen Kernaussagen zur europäischen Sicherheit der Konflikt zwischen der Ukraine und Russland bereits 2014 obsolet gemacht hat, endlich durch ein aktuelles Dokument ersetzen. Zwar sind die Strategischen Konzepte der NATO seit Ende des Kalten Krieges zunehmend zu einem Mittel öffentlicher Kommunikation der NATO geworden und sollten deshalb nicht überwertet werden, doch gibt die Art und Weise, in der die Bündnispartner bestimmte Themen artikulieren und hierarchisieren, Aufschluss über das gegenwärtige Selbstverständnis der Allianz.

Über die Schwerpunkte des neuen Konzepts lässt sich gegenwärtig nur spekulieren. Eines aber ist sicher: dem Klimawandel, dem militärischen Verständnis von Klimasicherheit und den Anpassungsbemühungen der NATO dürfte viel Raum gewidmet werden. Bereits das Strategische Konzept von 2010 wies erstmals auf die Verschärfung von Sicherheitsrisiken durch den Klimawandel hin, blieb jedoch folgenlos. Zum einen deshalb, weil es nicht detailliert verhandelt, sondern maßgeblich vom damaligen Generalsekretär Rasmussen entworfen worden war. Aufgrund dieses ungewöhnlichen Verfahrens (no line-by-line drafting by Allies) war der Grad an ownership der Natio-

Anmerkung: Die Verfasser geben ausschließlich ihre persönliche Meinung wieder.

\footnotetext{
*Kontakt: Rene Heise, Stabsoffizier in der Emerging Security Challenges Division im Internationalen Stab der NATO, Brüssel; E-Mail: Heise.Rene@hq.nato.int Michael Rühle, Leiter der Hybrid Challenges and Energy Security Section im Internationalen Stab der NATO; E-Mail: ruhle.michael@hq.nato.int
}

nen an dem Dokument nicht besonders ausgeprägt. Zum anderen verspürten manche Verbündete wenig Neigung, sich auf ein Thema einzulassen, welches das delikate Gleichgewicht der geopolitischen Interessen in bestimmten Regionen (z. B. im Hohen Norden) stören oder zu einer kontroversen Debatte über nationale Umwelt- oder Energiepolitik ausarten könnte. Die Ambivalenz einiger Verbündeter beim Thema Klimawandel spiegelte sich auch in ihrer begrenzten Bereitschaft, im Rahmen des NATO-Wissenschaftsprogramms Forschungsprojekte zu Klima- und Umweltthemen zu fördern. Die NATO, so das gängige Argument, sei für dieses Thema nicht das geeignete Gremium.

Einige Jahre später änderte sich die Haltung der NATO. Auf Grundlage moderner Klimamodelle und -szenarien sowie der wachsenden internationalen Anerkennung hochrangiger wissenschaftlicher Studien - allen voran die Berichte des International Panel on Climate Change (IPCC) - entwickelte sich ein zunehmend sachorientierter Austausch unter den Verbündeten. ${ }^{1}$ Ein von Dänemark und Litauen gemeinsam verfasstes Papier, das den Versuch eines Brückenschlags zwischen Umwelt- und Energiesicherheit unternahm, führte im Februar 2014 zur Verabschiedung des Green Defence Framework. Dieses listete zahlreiche Vorschläge zur Verringerung des Treibstoffverbrauchs in den Streitkräften sowie zur Einführung „grüner“ Standards in NATO-Einrichtungen auf. ${ }^{2}$ Der Enthusiasmus der Nationen hielt sich indes in Grenzen. Die zurückhaltenden Formulierungen des Dokuments ließen deutlich erkennen, dass viele Verbündete sich nicht vor-

1 Die Einordnung historischer Klimaschwankungen erfolgte in den letzten Jahrzehnten systematischer und gezielter, wodurch eine breite politische Akzeptanz erreicht wurde. Unterschiedliche Interpretationen der Signale der Erderwärmung - z. B. kurzfristige Temperaturschwankungen, historische Bahnänderungen der Erde, Sonnenaktivitätszyklen, Vulkanausbrüche - führten anfangs zu wissenschaftlichen Kontroversen, die jedoch zunehmend einem Konsens wichen, der die starke globale Erderwärmung auf Grundlage der anthropogenen Verstärkung des Treibhauseffekts als Tatsache akzeptiert und nach raschem Handeln verlangt.

2 NATO Green Defence Framework; siehe auch Rühle 2020. 
behaltlos auf das Thema einlassen wollten. Allzu viele Vorschläge waren im Konjunktiv formuliert und entfalteten daher keine Bindewirkung. Das geänderte Sicherheitsumfeld durch die Ukraine-Krise, die im März 2014 mit der russischen Annexion der Krim ihren Höhepunkt erreichte, tat ein Übriges, um das Green Defence Framework politisch zu marginalisieren: infolge der durch das russische Verhalten ausgelösten Rückbesinnung der NATO auf ihre Kernaufgabe der kollektiven Verteidigung mutete das Green Defence Framework wie eine Ablenkung vom Bündniszweck an, die man sich nicht mehr leisten zu können glaubte. Eine Einschränkung operativer militärischer Fähigkeiten durch klimapolitische Restriktionen schien nun erst recht unzeitgemäß.

Dennoch diskutierte man in den folgenden Jahren immer wieder die sicherheitspolitischen Konsequenzen des Klimawandels, unter anderem anhand interner Analysen der politischen und militärischen Stäbe und in informellen Debatten der NATO-Botschafter. Gleichzeitig versuchte ein vom NATO-Wissenschaftsprogramm gefördertes Smart-Energy-Projekt, den Verbrauch von Brennstoffen in abgelegenen Stützpunkten bzw. Feldlagern durch eine Integration von Wind- und Solarenergie zu verringern und damit zugleich den Ausstoß von Treibhausgasen zu senken. ${ }^{3}$ Ein systematischer Einstieg der NATO in das Klima-Dossier gelang jedoch erst im Sommer 2020, als Generalsekretär Stoltenberg seine Politische Planungseinheit beauftragte, die potenzielle Rolle der NATO in Fragen der Klimasicherheit zu analysieren. Als die Wahl Joe Bidens zum neuen amerikanischen Präsidenten eine Kehrtwende der Klimapolitik der USA erwarten ließ und der im November 2020 erschienene Bericht der von Stoltenberg installierten Reflection Group (NATO 2030: United for a New Era $)^{4}$ eine verstärkte Betrachtung des Klimawandels durch die NATO-Allianz befürwortete, schien die Zeit endlich reif für eine umfassende Auseinandersetzung der NATO mit den Folgen des Klimawandels.

3 NATO Smart Energy Team (SENT): Comprehensive Report 2015. 4 NATO 2030: United for a New Era. Analysis and Recommendations of the Reflection Group appointed by the NATO Secretary General, 25 November 2020.

\section{Der globale Klimawandel: sicher- heitspolitische und militärische Auswirkungen}

Die sichtbarsten Auswirkungen des Klimawandels sind heute die häufigeren und stärkeren ausgeprägten Hitzewellen (meist gekoppelt mit verheerenden Waldbränden), der Anstieg des Meeresspiegels, der Meereisrückgang (in der Arktis um $40 \%$ seit 1979) sowie veränderte Niederschlagsmuster (Überflutungen und Dürreperioden). Darüber hinaus erscheint immer fraglicher, ob sich bestimmte ökologische Systeme (Arktis, Amazonas-Regenwald, Korallenriffe) überhaupt noch regenerieren können. Was vor Jahrzehnten als Utopie anmutete, wird auf Grundlage von Satellitenvergleichsmessungen mit stetig wachsender Präzision und verbesserten Klimamodellen (dank schnellerer Hochleistungsrechner) zunehmend Realität und damit zu einem Faktor, der auch in militärstrategischen Planungen seinen Niederschlag finden muss. Es ist davon auszugehen, dass die Entwicklungen des Klimasystems eine kritische Schwelle (tipping point, deutsch: Kipppunkt) überschreiten werden. Die Folgen wäre neben der Änderung großräumiger atmosphärischer und ozeanischer Zirkulationsmuster auch ein Verlust an Biodiversität, zum Beispiel massive Gleichgewichtsveränderungen bis hin zum Kollaps des empfindlichen arktischen Ökosystems. Eine solche Entwicklung ginge einher mit drastischen Engpässen in der globalen Nahrungsmittelversorgung. In bekannter Form wären Kompensationsoptionen durch die Gesellschaften dann möglicherweise nicht mehr gegeben.

Die Komplexität der Prozesse im System „Ozean-Atmosphäre" und eine Betrachtung der wissenschaftlichen Fakten aus militärisch-operativer Sicht erfordern eine permanente Anpassung der Risikobewertung durch das Bündnis. Dies ist umso mehr geboten, als manche der gegenwärtig zu beobachtenden klimatischen Entwicklungen unumkehrbar sind. Global wird der Ausstoß von $\mathrm{CO}_{2}$ und anderen Treibhausgase aller Voraussicht nach in den nächsten 30 Jahren weiter ansteigen. ${ }^{5}$ Das Szenario einer Erwärmung der globalen Oberflächentemperatur von $3^{\circ} \mathrm{C}$ im weiteren Verlauf des Jahrhunderts erscheint zunehmend realistisch. Damit einhergehende Veränderungen sind wegen der hohen Verweildauer diverser Treibhausgase (bei $\mathrm{CO}_{2}$ über Jahrhunderte), so der aktuelle IPCC-Sachstandsbericht, zudem irreversibel. ${ }^{6}$ Bei der Betrachtung der Indikatoren für den Klimawandel stellt sich

5 United States Energy Information Agency 2019. 6 International Panel on Climate Change 2021. 
auch die Frage der militärstrategischen Risikobewertung völlig neu. ${ }^{7}$ Ziel muss es sein, die Allianz zu befähigen, mit derartigen Unsicherheiten umgehen zu können, ohne ihren Kernauftrag zu gefährden.

Die sicherheitspolitischen Konsequenzen der Erderwärmung sind unschwer zu erkennen. ${ }^{8}$ Der Anstieg des Meeresspiegels (höchste Anstiegsraten in den letzten 3.000 Jahren), verbunden mit Landverlust (insbesondere bei dichtbesiedelten Küstenregionen), bei gleichzeitiger Versteppung weiter Landstriche könnte Migrationsströme auslösen, Dispute um bewohnbares Land bzw. landwirtschaftliche Nutzflächen könnten sich militärisch entladen und die Zunahme extremer Wetterereignisse wie Stürme oder Feuersbrünste zu humanitären Katastrophen führen, in denen den Streitkräften als first responder eine wichtige Rolle zukäme. Mehr noch: Die schmelzenden Eiskappen am Nordpol eröffnen neue Seewege (Nordost- und Nordwestpassage) und schaffen damit nicht nur neue wirtschaftliche Perspektiven, sondern auch neue Gebiete potenzieller geopolitisch-militärischer Konkurrenz. Bei dieser kann es um den Zugang zu Rohstoffen gehen, um die Erweiterung von Hoheitsgebieten oder um Versuche der Neubestimmung von Festlandsockeln. Zahlreiche Staaten haben folglich den Klimawandel zu einem vorrangigen Problem der nationalen Sicherheit erklärt.

Der Klimawandel beeinflusst bereits heute die Planung und Durchführung militärischer Operationen. ${ }^{9}$ So hat der veränderte Salzgehalt des Meerwassers im Golf von Aden die Leistung der Turbinen britischer Fregatten verringert. Die deutschen Truppen in Afghanistan hatten schon seit Jahren damit zu kämpfen, dass die Sommerhitze im gegliederten Gelände bzw. in Höhenlagen des Hindukusch die Startleistung - und somit die Nutzlast - von Hubschraubern und Transportflugzeugen verringerte. Die Häufung und zunehmende Intensität von Sand- und Staubstürmen erschwerte überdies die Planung und Durchführung von taktisch-militärischen Flügen. Die steigenden Temperaturen im Irak wiederum haben die dort stationierten west-

\footnotetext{
7 Die globale Erdoberflächentemperatur ist nur einer von mehreren Indikatoren für den Klimawandel, auch wenn sie in der Klimadebatte nahezu im Mittelpunkt steht. Eigentlich ist der Wärmeinhalt der Ozeane ein besserer Indikator für die globale Erwärmung als die (kurzzeitig) stärker schwankende Lufttemperatur. Angesichts der Komplexität der Prozesse bei den verschiedenen Elementen des Klimasystems (Atmosphäre, Ozeane, Land- und arktische Gebiete, Kopplungseffekte mit der Biosphäre) konzentrierte sich der öffentliche Diskurs fast ausschließlich auf das 1,5-Grad-Limit für die globale Erwärmung der Lufttemperatur. Diese vereinfachte Formel wird dem Prozess und der Physik des Klimawandels jedoch nicht völlig gerecht. 8 Galgano 2019.

9 Heise 2021.
}

lichen Soldaten immer wieder an die Grenze ihrer Leistungsfähigkeit gebracht, da ihre modernen Kampfanzüge nicht für Temperaturen um 50 Grad oder höher ausgelegt sind. In bestimmten Regionen wird im Hitzestress die physiologische Anpassungsgrenze (Thermoregulation) des Menschen überschritten werden. Eine Herausforderung stellen ferner in diesem Zusammenhang die Überhitzung empfindlicher Flugzeug- und Waffenelektronik sowie die erforderliche energieintensive Kühlung der Infrastruktur in Feldlagern und Flugplätzen dar. Außerdem gefährdet der ansteigende Meeresspiegel küstennahe Militärbasen; Dutzende amerikanischer Marinestützpunkte sind weltweit akut davon betroffen. Aber auch militärische Installationen und Truppenübungsplätze im Inland sind gegen die Folgen des Klimawandels (insbesondere Waldbrände) nicht immun. ${ }^{10}$ Der Umfang der durch extreme Wetterereignisse verursachten Schäden an Militärstützpunkten wächst stetig. Die Herausforderungen von Klimawandel und Veränderungen in den oberen Atmosphärenschichten werden sich ebenfalls bei militärischen Aktivitäten in der neuen Dimension Weltraum zeigen. ${ }^{11}$

Ein weiterer Grund, weshalb das Militär die Klimadiskussion aufmerksam verfolgen sollte, ist die Dekarbonisierung der westlichen Volkswirtschaften, die besonders deutlich in den Plänen der Europäischen Union zum Ausdruck kommt, Europa bis 2050 „klimaneutral“ zu machen. Die graduelle Abkehr von fossilen Brennstoffen im zivilen Bereich wird früher oder später Auswirkungen auf die Streitkräfte haben. Zwar kann das Militär mittels Ausnahmeregelungen vermutlich länger an fossilen Brennstoffen festhalten, es wird im Falle einer Elektrifizierung des Transportwesens letztendlich aber eine Elektrifizierung des militärischen Fuhrparks nicht umgehen können. Dies dürfte zumindest für diejenigen Fahrzeuge gelten, die nicht - wie etwa Panzer - unmittelbar für Kampfeinsätze vorgesehen sind. Für letztere ließen sich neue Treibstoffe auf der Grundlage von Wasserstoff-Brennstoffzellen finden - eine derzeit teure und energieintensive Lösung (Wasserstoffherstellung), die man in größerem Umfang bislang nur in U-Booten erprobt (siehe unten). Obzwar die Abkehr von fossilen Brennstoffen in den Streitkräften wahrscheinlich später erfolgen wird als im zivilen Bereich, zeichnen sich hier neue Realitäten ab, die auch die Streitkräfte nicht ignorieren können. In einem ersten Schritt werden sich in naher Zukunft vermutlich sogenannte Technologiemix-Systeme durchsetzen und einen Teilbeitrag zur Reduzierung des militärischen „CO2-Fußabdrucks“ leisten.

10 United States Department of Defense 2019. 11 Heise 2021. 


\section{Die künftige Rolle der NATO bei der Klimasicherheit}

Der fortschreitende Klimawandel und die damit einhergehenden Risiken führten im März 2021 zur Verabschiedung einer Climate Change and Security Agenda durch die NATO-Außenminister. Sie definiert erstmals klar die künftige Rolle der NATO bei der Klimasicherheit. Kaum drei Monate später vereinbarten die Staats- und Regierungschefs des Bündnisses auf ihrem Brüsseler Gipfeltreffen einen Climate Change and Security Action Plan, der eine detaillierte Auflistung der einzelnen Maßnahmen enthält, die das Bündnis unter den Rubriken awareness, adaptation, mitigation und outreach zusammenfasste. ${ }^{12}$ In einem parallel verlaufenden Verhandlungsprozess einigten sich die NATO-Botschafter zudem auf eine Reihe von Vorschlägen, die der Generalsekretär in eigener Regie unter dem Titel NATO 2030 propagiert hatte. ${ }^{13}$ Einer dieser Vorschläge sieht eine stärkere Aufgabe in der Klimasicherheit, mit dem Schwerpunkt auf Verringerung der Treibhausgase in den NATO-Streitkräften.

Die künftige Rolle der NATO bei ihrer Auseinandersetzung mit dem Klimawandel beginnt bei einer neuen Form der Lageeinschätzung. In Zukunft sollen jährliche Berichte den Einfluss der globalen Erwärmung auf das strategische Umfeld der NATO sowie auf ihre konkreten Einsätze festhalten. Ebenso müsse das Bündnis die Auswirkungen des Klimawandels in ihre Risikoanalysen sowie in ihre Bewertungen der „Resilienz“ einzelner Verbündeter und Partnerstaaten einbeziehen. Die Wissenschaftsprogramme der NATO, die schon seit Jahrzehnten Forschungen zu Klimaund Umweltsicherheit fördern, seien noch stärker auf Fragen des Klimawandels und der sich daraus ergebenden neuen Herausforderungen auszurichten.

Darüber hinaus soll die NATO Klimafragen künftig in das gesamte Spektrum ihrer Aktivitäten einbeziehen, so etwa in Verteidigungsplanung, zivile Notfallplanung, Standardisierung, militärische Übungen und Katastrophenhilfe. Die Beschaffungspolitik müsse bei den Anforderungen an die zukünftigen militärischen Fähigkeiten der NATO berücksichtigen, dass der Klimawandel das strategische Umfeld schnell verändern und immer extremere Umweltbedingungen schaffen wird. Zugleich seien die Auswirkungen des Klimawandels auf die Abschreckungs- und Verteidigungsfähigkeit der NATO zu unter-

12 NATO Climate Change and Security Action Plan, 14. Juni 2021. 13 NATO 2030, Fact Sheet, Juni 2021. suchen, wozu die Zuführung von Verstärkungen und die militärische Mobilität gehört.

Schließlich will die NATO auch den Dialog mit anderen Institutionen sowie mit ihren zahlreichen Partnerstaaten suchen. Dies gilt vor allem für die Vereinten Nationen und die EU, denen wesentliche Führungsaufgaben bei der Bekämpfung des Klimawandels zukommen und deren Beschlüsse die künftige Politik der NATO zumindest indirekt beeinflussen. Bei den Beziehungen zu den Partnerstaaten, von denen viele vom Klimawandel aktuell stärker betroffen sind als die Verbündeten, dürfte speziell die wissenschaftliche Zusammenarbeit im Vordergrund stehen. Hier könnten die Entwicklung und Nutzung von Sensoren zur Erfassung militärisch relevanter Wetter- und Klimadaten sowie der Datenaustausch - insbesondere in datenarmen Regionen - einer der nächsten Schritte sein. Gerade moderne Verfahren der künstlichen Intelligenz benötigen in diesem Kontext große Datenmengen, und die Nutzung innovativer Datenorganisationsformen (Datenwürfel) zur mehrdimensionalen Analyse könnte große Fortschritte ermöglichen. ${ }^{14}$ So ließen sich aus Veränderungsanalysen, gekoppelt mit taktischen Gefechtsmodellen, neue Optionen für operative Planungen entwickeln. Ebenso sind die Erfahrungen, die Verbündete bei der Steigerung der Energieeffizienz in ihren Streitkräften machen, interessant für manche Partnerstaaten. Überdies will man mit der Zivilgesellschaft verstärkt ins Gespräch kommen eine von der NATO initiierte jährliche Großveranstaltung zum Thema Klimawandel und Sicherheit soll bereits 2022 erstmals stattfinden.

\section{Mitigation als größte Herausforderung}

Das wohl anspruchsvollste Vorhaben des neuen NATOPlans ist es, einen aktiven Beitrag zur Verlangsamung (mitigation) des Klimawandels zu leisten. Hierfür wird die NATO zunächst die analytischen Grundlagen entwickeln müssen, um Treibhausgase militärischer Aktivitäten und Installationen zu messen. Damit wäre eine Vergleichbarkeit nationaler Streitkräfteemissionen hergestellt, die

14 Ein Beispiel ist Cube4Sec: Big Earth Datacube Analytics for Transnational Security and Environment Protection. Dieses Projekt soll demonstrieren, wie Daten zu dynamischen Erkenntnissen über natürliche wie auch vom Menschen verursachte Bedrohungen jeglicher Art beitragen können. Hierfür werden boden-, weltraum- und luftgestützte Quellen in Echtzeit kombiniert. 
den Bündnisstaaten bei der Formulierung - freiwilliger Emissionsziele helfen soll. Im Endeffekt, so die Hoffnung der Verbündeten, soll sich die Sammlung vergleichbarer Daten zum militärischen Energieverbrauch positiv auswirken auf künftige nationale Innovations- und Investitionsentscheidungen, die eine Entwicklung energieeffizienterer Streitkräfte fördern.

Der Anspruch der Verbündeten, die TreibhausgasEmissionen ihrer Streitkräfte zu verringern, wirft unweigerlich die Frage nach der Machbarkeit auf. Unbestritten schafft die Energiewende, die durch Strom und klimaneutrale Kraftstoffe zur Dekarbonisierung beitragen soll, neue Risiken. So verlangen etwa der Transport und die Integration von Wasserstoff in den militärischen Energiemix ein völlig anderes Sicherheitsmanagement als traditionelle Treibstoffe. Zudem gehen militärische Konflikte mit neuen technischen Herausforderungen einher, sei es beim Schutz der Produktionsanlagen oder dem Transport und letztendlich im Einsatz. Auch ist die Energiedichte der bekannten erneuerbaren Energien geringer als die von klassischen fossilen Treibstoffen wie etwa Diesel. Für ein Verteidigungsbündnis steht allerdings die Aufrechterhaltung seiner militärischen Kampfkraft stets an erster Stelle. Eine Reduktion der Emissionen, beispielsweise durch die graduelle Abkehr von fossilen Treibstoffen, macht also nur Sinn, wenn sie die militärische Leistungsfähigkeit der Streitkräfte nicht beeinträchtigt oder - besser noch sogar erhöht. Versuche unter den nationalen Streitkräften vieler NATO-Staaten zeigen, dass ein Senken der Emissionen und ein Steigern der militärischen Leistungsfähigkeit sich nicht zwingend widersprechen müssen.

Ein bekanntes Beispiel für ein solches Win-win-Ergebnis ist die Nutzung innovativer Brennstoffzellen als Antriebstechnologie für U-Boote, die operative Vorteile durch geräuscharme, lange und tiefe Tauchgänge ermöglicht. Brennstoffzellen werden auch bei Militärkraftfahrzeugen, Hubschraubern, Flugzeugen und Panzern getestet. Batterielösungen, gekoppelt mit elektrischen Antriebstechnologien, erweisen sich hingegen durch das hohe Gewicht und die geringe Energieeffizienz als nur begrenzt nutzbar, insbesondere in den hohen Breiten (mit niedrigen Lufttemperaturen). Mehr Erfolg versprechen dagegen taktische Drohneneinsätze in Gebirgsregionen mit leistungsfähigen elektrischen Antriebssystemen. Bei diesen ist die technologische Anpassung an Höhenbereiche mit geringer Luftdichte unkompliziert und beherrschbar, zumal Verbrennungsmotoren von Flugzeugen und Hubschraubern in Höhen oberhalb von drei Kilometern schnell ihre Leistungsgrenze erreichen. Die NATO-Verbündeten haben den Generalsekretär beauftragt, die Machbarkeit eines Netzero-Szenarios für NATO-eigene Installationen bis $2050 \mathrm{zu}$ untersuchen. ${ }^{15}$ Das berührt jedoch nicht die Streitkräfte der Mitgliedstaaten, da die Reduktion militärischer Treibhausgase ihre freiwillige Entscheidung bleibt. Allein dies zeigt, dass es beim Thema mitigation auch darum gehen wird, überzogene Erwartungen zu dämpfen - umso mehr, weil Streitkräfte, vor allem Luftstreitkräfte, ungeachtet zahlreicher grüner Initiativen auch absehbar weiterhin große Mengen an fossilem Brennstoff verbrauchen werden.

\section{Klimawandel im neuen Strategischen Konzept der NATO}

Bis zur Vorstellung des neuen Strategischen Konzepts auf dem NATO-Gipfel in Spanien im Sommer 2022 werden zahlreiche im Climate Change and Security Action Plan vorgesehenen Schritte bereits unternommen worden sein. Hierzu zählt ein Bericht des Generalsekretärs über die Auswirkungen des Klimawandels auf Operationen und Installationen der NATO ebenso wie die stärkere Berücksichtigung von Klimafaktoren bei den diversen Analysen der Resilienz der Verbündeten. Auch das NATO-Wissenschaftsprogramm dürfte bis zum Sommer 2022 mehrere neue Projekte umfassen, die sich mit den sicherheitspolitischen Folgen des Klimawandels beschäftigen, insbesondere mit dem Erfassen von explizit militärisch relevanten Wetter- und Klimadaten, verbunden mit der Bewertung aktueller Klimaprojektionen. Anders als das Konzept von 2010, das weder eine politische noch eine militärische Klimaagenda generierte, wird sein Nachfolger bei diesem Thema also bereits auf einem institutionellen acquis aufbauen können.

Die Herausforderung für das neue Strategische Konzept dürfte folglich nicht in der Formulierung einer NATOKlimaagenda liegen, die inzwischen existiert, sondern im Stellenwert, den die Allianz dem Thema einräumt. Des Weiteren werden die Verbündeten bei den Verhandlungen über das neue Strategische Konzept vermutlich mit der Frage ringen, wie ambitioniert sie das Ziel mitigation angehen wollen. Beim ausschließlich mit Klimafragen befassten Action Plan war es nicht allzu schwierig, weitreichende Absichtserklärungen hinsichtlich der Ver-

15 So heißt es in der entsprechenden Erklärung: “Leaders committed to significantly reduce greenhouse gas emissions from military activities and installations. They invited the Secretary General to develop a concrete and ambitious target for the reduction of greenhouse gas emissions by the NATO political and military structures and facilities, and to assess the feasibility of reaching net zero emissions by 2050." NATO 2030 Fact Sheet, ibid. 
ringerung militärischer Treibhausgase $\mathrm{zu}$ formulieren. In einem Dokument hingegen, das die gesamte NATOAgenda abdecken muss und in dem die Verbündeten vermutlich unterschiedliche Schwerpunkte setzen werden, könnte das Bekenntnis zu mitigation weniger umfassend ausfallen als beim Erhalt der Abschreckungs- und Verteidigungsfähigkeit der NATO. Gerade angesichts dynamischer Klimaveränderungen muss die Allianz ihren Fokus weiterhin darauf richten, militärische Fähigkeiten zu bewahren, die ihren Auftrag selbst unter extremen Umweltbedingungen erfüllen können. Das erfordert, dass sich die einschlägigen NATO-Gremien stetig mit klimatischen Veränderungen und notwendigen Anpassungen befassen. Da Wissenschaft immer nur Erkenntnis auf Zeit ist, muss das Bündnis auch auf unvorhergesehene Entwicklungen umgehend reagieren können, ohne seinen Kernauftrag zu gefährden. Um dies zu erreichen, müssen die jeweiligen Fähigkeiten in der Kommando- und Streitkräftestruktur der NATO entsprechend abgebildet sein.

Eines aber ist sicher: Die Beschäftigung der NATO mit dem Dossier Klimasicherheit ist kein lediglich dem öffentlichen Erwartungsdruck geschuldeter kurzlebiger Aktionismus, sondern ein fester Bestandteil der künftigen NATO-Agenda. Dafür sorgen nicht nur der Climate Change and Security Action Plan, sondern insbesondere auch die zunehmend gravierenden Auswirkungen der globalen Erwärmung. Der Zusammenhang von Sicherheit und Klimafragen wird immer deutlicher erkennbar. Ein neues Strategisches Konzept sollte ihn schonungslos, jedoch ohne Alarmismus beschreiben. Vor allem aber darf die Diskussion um den militärischen Beitrag bei der Verringerung von Treibhausgasen nicht in eine Stigmatisierung von Streitkräften als Mitverursacher des Klimawandels abgleiten. Klimaschutz und militärische Sicherheit sind von existenzieller Bedeutung und dürfen nicht gegeneinander ausgespielt werden.

\section{Literatur}

Galgano, Francis, Hrsg. (2019): The Environment-Conflict Nexus: Climate Change and the Emergent National Security Landscape. Wiesbaden: Springer e-book; https://doi. org/10.1007/978-3-319-90975-2_3

Heise, Rene (2021): NATO is responding to new challenges posed by climate change, NATO Review, 1 April 2021; https://www.nato. int/docu/review/articles/2021/04/01/nato-is-responding-tonew-challenges-posed-by-climate-change/index.html

International Panel on Climate Change (2021): Sixth Assessment Report, Climate Change 2021: The Physical Science Basis, the Working Group I. New York: IPCC; https://www.ipcc.ch/report/ ar6/wg1/

Rühle, Michael (2020): Scoping NATO's Environmental Security Agenda Rom: NATO Defense College, Policy Brief No. 19

\section{Dokumente der NATO und einzelner Regierungen}

NATO Climate Change and Security Action Plan, 14 June 2021; https://www.nato.int/cps/en/natohq/official_texts_185174. $\mathrm{htm}$ ?selectedLocale $=$ en

NATO Green Defence Framework, February 2014; https:// natolibguides.info/ld.php?content_id=25285072

NATO Smart Energy Team (SENT): Comprehensive Report, 6 May 2015; https://natolibguides.info/ld.php?content_id=18110194

NATO 2030 Fact Sheet, June 2021; https://www.nato.int/ nato_static_fl2014/assets/pdf/2021/6/pdf/2106-factsheetnato2030-en.pdf

NATO 2030: United for a New Era. Analysis and Recommendations of the Reflection Group appointed by the NATO Secretary General, 25 November 2020: https://www.nato.int/nato_static_fl2014/ assets/pdf/2020/12/pdf/201201-Reflection-Group-FinalReport-Uni.pdf

United States Department of Defense (2019): Report on Effects of a Changing Climate to the Department of Defense; https://media. defense.gov/2019/Jan/29/2002084200/-1/-1/1/CLIMATECHANGE-REPORT-2019.PDF

United States Energy Information Administration EIA (2019): International Energy Outlook 2019 with projections to 2050. Washington, DC: US-EIA; https://www.eia.gov/outlooks/ archive/ieo19/pdf/ieo2019.pdf 